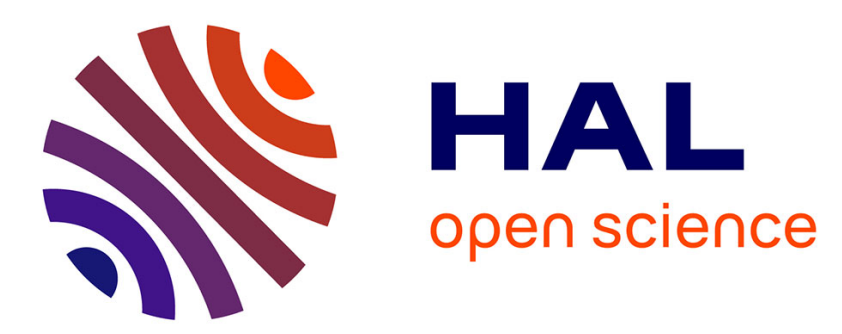

\title{
Déploiement du e-learning dans le cadre de la formation en entreprise: quel rôle de l'environnement social dans l'engagement des salariés?
}

\author{
Emmanuel Baudoin, Loubna Tahssain-Gay
}

\section{- To cite this version:}

Emmanuel Baudoin, Loubna Tahssain-Gay. Déploiement du e-learning dans le cadre de la formation en entreprise: quel rôle de l'environnement social dans l'engagement des salariés?. Recherches en sciences de gestion, 2013, 97, pp.109-126. 10.3917/resg.097.0109 . hal-02899178

\section{HAL Id: hal-02899178 \\ https://hal.science/hal-02899178}

Submitted on 14 Jul 2020

HAL is a multi-disciplinary open access archive for the deposit and dissemination of scientific research documents, whether they are published or not. The documents may come from teaching and research institutions in France or abroad, or from public or private research centers.
L'archive ouverte pluridisciplinaire HAL, est destinée au dépôt et à la diffusion de documents scientifiques de niveau recherche, publiés ou non, émanant des établissements d'enseignement et de recherche français ou étrangers, des laboratoires publics ou privés. 
revue Recherches en Sciences de Gestion-Management Sciences-Ciencias de Gestión, n97, p.

\title{
Déploiement du e-learning dans le cadre de la formation en entreprise : quel rôle de l'environnement social dans l'engagement des salariés ?
}

\author{
Emmanuel Baudoin \\ Professeur Associé \\ Télécom Ecole de Management \\ Loubna Tahssain-Gay \\ Professeur Associé \\ Groupe Sup de co La rochelle \\ Pôle RH\&M
}

Le e-learning poursuit sa progression dans le paysage de la formation en entreprise. Cet ensemble de modalités multimédias de formation peut être considéré comme révolutionnaire, et impliquant des changements de méthodes de formation qui ne sont pas neutres $(P$. Gil, 2000). La question de la conduite du changement s'avère capital pour ce type de projets (B. Bachimont et al. 2002). Cette progression s'accompagne en effet de taux d'abandon qui peuvent rester important (C. Baujard, 2007, 2008; E. Bernardin, 2006; K. Frankola, $2001 ; M-D$. Lytras et A. Pouloudi, 2001) et remettre en cause l'efficacité du e-learning dans les dispositifs de formation. Très souvent, ces projets sont mal gérés par les entreprises et engendrent ainsi des pertes appelés "coûts cachés" (H. Savall et V. Zardet, 2003). Les modes de déploiement des parcours e-learning auprès des salariés dans les grandes entreprises deviennent alors un enjeu primordial pour tenter de faire face à cette situation, le but étant alors de favoriser l'engagement des salariés dans leurs parcours elearning. 
Quelles sont les différentes dimensions du déploiement d'un projet e-learning? Comment influent-elles sur l'engagement des salariés?

Pour répondre à ces questions, nous avons mené quatre études de cas enchâssées dans un même contexte organisationnel, celui d'un constructeur automobile international. Quarante-huit entretiens avec des salariés ont été conduits. Les résultats ont permis de relever que les dimensions technologiques et pédagogiques d'un déploiement constituent le socle de la réussite de celui-ci. La dimension organisationnelle, avec l'environnement social, permet d'individualiser et d'adapter le déploiement aux salariés.

Mots-clés : formation continue, e-learning, conduite du changement, environnement social.

E-learning continues to grow in the landscape of training. This set of multimedia training methods can be considered revolutionary, involving changes to training methods that are not neutral (Gil, 2000). The subject matter of change management is crucial for this type of project (C. Baujard, 2007, 2008; E. Bernardin, 2006; K. Frankola, 2001; M-D. Lytras et A. Pouloudi, 2001) and question the effectiveness of e-learning in training devices. Very often, these projects are poorly managed by companies; thus, it generates losses called "hidden costs" (H. Savall and V. Zardet, 2003). The e-learning deployment methods for employees in large companies become a key issue in an attempt to cope with this situation, the goal is then to foster employee engagement in their e-learning courses.

What are the different dimensions of the deployment of an elearning project? How do they affect employees' engagement?

To answer these questions, we conducted four case studies within the same organizational context, which is an international automobile manufacturer. Forty-eight interviews with employees were conducted. The achieved results allowed us to identify that technological and pedagogical dimensions of a deployment are the foundation of the success of it. The organizational dimension, the social environment, allows individualizing and tailoring the deployment to employees.

Key-words: continuing education, e-learning, change management, social environment. 
El e-learning continúa su progresión en el paisaje de la formación en las empresas. Esta modalidad formativa multimedia puede ser considerada como revolucionaria e implicar cambios en los métodos de formación que no son neutros (Gil, 2000). Es por ello que, en este tipo de proyectos, la problemática del acompañamiento al cambio puede aparecer como capital (Bachimont y al. 2002) pues dicha progresión puede también verse acompañada de una tasa de abandono importante (C. Baujard, 2007, 2008; E. Bernardin, 2006; K. Frankola, 2001; M-D. Lytras y A. Pouloudi, 2001) y, así, poner en cuestión la eficacia de los dispositivos de formación continua online. Muy a menudo, estos proyectos de formación son mal gestionados por las empresas generando pérdidas, son los denominados "costos ocultos" (H. Savall y V. Zardet, 2003). En consecuencia, la forma en que van a desarrollarse los programas de e-learning para los asalariado(a)s en las grandes empresas se convierte en un desafio primordial para intentar hacer frente a esta situación, puesto que el objetivo principal sería el de favorecer la implicación de los asalariado(a)s en sus itinerarios de formación a distancia. ¿Cuáles son las dimensiones de un proyecto e-learning? ¿De qué manera influyen en la implicación de los asalariado(a)s? Para responder a estas preguntas hemos llevado a cabo cuatro estudios de caso en un mismo contexto organizativo, el de un fabricante internacional de automóviles. Hemos entrevistado a cuarenta y ocho asalariado(a)s y los resultados han permitido poner de relieve que las dimensiones tecnológica y pedagógica de un programa de e-learning constituyen la base del éxito del mismo. La dimensión organizativa, teniendo en cuenta el entorno social, permitiría individualizarlo $y$ adaptarlo mejor a cada caso.

Palabras claves: formación continua, e-learning, acompañamiento al cambio, entorno social".

\section{Introduction}

Le e-learning s'est ancré dans le paysage de la formation en entreprise depuis plus d'une décennie. Cette tendance a été confirmée par l'enquête menée par l'observatoire CEGOS en 2013. 
Cet ensemble de modalités multimédias de formation peut être considéré comme innovant, et impliquant des changements de méthodes de formation qui ne sont pas neutres (P. Gil, 2000). D'un côté, l'utilisation du e-learning en entreprise vise à offrir des contenus uniformisés, disponibles en tout temps et à tout moment pour l'ensemble des salariés d'une entreprise (C. Baujard, 2004, 2005, 2007 ; N. Beamish et al., 2002 ; D. Beckett et al., 2002 ; M. Favier et al. 2004). L'enjeu est d'améliorer la qualité pédagogique des contenus délivrés aux salariés et favoriser la disponibilité de ces mêmes contenus. D'un autre côté, l'utilisation du e-learning peut modifier les habitudes de formation des salariés : modification des lieux et temps de formation (M. Kalika, 2001). Cette progression du e-learning dans les dispositifs de formation s'accompagne en effet de taux d'abandon qui peuvent rester important (C. Baujard, 2007, 2008 ; E. Bernardin, 2006 ; K. Frankola, 2001 ; M.D. Lytras et A. Pouloudi, 2001). La question de la conduite du changement s'avère dès lors capital pour ce type de projets (B. Bachimont et al. 2002) notamment pour les chefs de projets e-learning ou les responsables formation. Les modes de déploiement des parcours e-learning auprès des salariés dans les grandes entreprises deviennent ainsi un enjeu primordial pour tenter de faire face à cette situation, le but étant alors de favoriser l'engagement des salariés dans leurs parcours e-learning (K.T. Upadhyaya, M. Debasis, 2013 ; G. Netteland, 2009). Au niveau académique, G. Piccoli et al. (2001), dans leur taxonomie de l'efficacité du e-learning, soulignent l'importance du taux de suivi des participants au même titre que d'autres dimensions comme l'apprentissage ou la satisfaction des participants.

Cet article s'articule autour de trois parties. Dans un premier temps, nous aborderons les trois dimensions du déploiement d'un projet e-learning: technologique, pédagogique et organisationnelle. Le rôle particulier de l'environnement social, à travers l'environnement organisationnel, sera mis en exergue. Dans un deuxième temps, nous présentons la méthodologie de recherche basée sur quatre études de cas enchâssées dans un même contexte organisationnel, celui d'un constructeur automobile international. 81 entretiens ont été conduits dont 48 avec des salariés. Tous ces salariés ont été réellement engagés dans leur parcours e-learning. Ils se sont effectivement connectés aux modules multimédias constituant leurs parcours et les ont suivi. Dans un troisième temps, les résultats sont présentés. Le rôle de l'environnement technologique sur l'engagement des salariés est analysé à travers l'utilité perçue, la facilité d'utilisation perçue et la qualité perçue. Cet environnement technologique est le résultat du 
déploiement technologique et pédagogique. Les résultats montrent que ces deux dimensions du e-learning constituent le socle d'un déploiement réussi. Les influences de l'environnement social ainsi que de l'environnement spatial et temporel sont ensuite étudiés. Elles sont les résultantes du déploiement organisationnel. Les résultats montrent que les salariés ne sont pas sensibles de la même manière à ces deux environnements et notamment l'environnement social. Certains salariés ont besoin d'un environnement social incitatif alors que d'autres ont besoin d'un environnement social de soutien. Cet environnement constitue, à notre sens, la clé de l'individualisation du déploiement du e-learning en entreprise.

\section{1. - Revue de littérature}

\subsection{Définition du e-learning}

Selon L. Marchand et N. Lauzon (2004) et E.T. Welsh et al. (2003), le e-learning recouvre un continuum de modalités pédagogiques en ligne. Modules multimédias d'autoformation, classes virtuelles, forums de discussion ou encore jeux de simulation (serious games en anglais) en sont quelques illustrations. Pour tenter de saisir la diversité des modalités du e-learning, la définition retenue dans ce travail est la suivante : un ensemble de modalités multimédias de formation en ligne qui vise des apprentissages spécifiques ( $T$. Bondarouk, H. Ruël, 2010 ; R.C. Clark, R.E. Mayer, 2008 ; R.E. DeRouin et al., 2005 ; D. Drillon, 2002 ; M. Favier, 2003 ; E. Salas et al., 2002 ; A-S A Taylor, P. Backlund, et L. Niklasson,, 2012).

Il est important de noter que ce travail se focalise sur une modalité particulière : les modules multimédias d'autoformation. Un module multimédia d'autoformation est défini comme une présentation séquencée d'informations sur un thème, sous forme d'images, de mots et de sons numérisés (R.C. Clark, R.E. Mayer, 2008). Ces contenus sont diffusés via l'intranet ou l'extranet de l'entreprise. Un ou plusieurs module(s) multimédia(s) d'autoformation portant sur un thème de formation constitue(nt) un parcours e-learning (M. Prat, 2008), autre notion importante de cette recherche.

\subsection{Dimensions du déploiement de parcours e-learning}


Plusieurs chercheurs se sont employés à décrire les différentes dimensions du déploiement du e-learning dans les entreprises. La majorité s'est intéressée à une ou deux dimensions particulière(s). Selon nous, A. Welle-Strand et T. Thune (2003) offrent la vision la plus complète.

\subsubsection{Dimension technologique : proposition d'une définition et présentation des attributs}

La technologie constitue la pierre angulaire du e-learning (G. Martin et al., 2003 ; A. Mitchell, S. Honore, 2007 ; D. Mueller, S. Strohmeier, 2010 ; D. Newton et al., 2002 ; R.H. Wild et al., 2002). L'utilisation de la technologie suppose en effet la réalisation des contenus multimédias et l'accès des utilisateurs finaux à ces mêmes contenus (T. Lau, 2003). Selon A.Welle-Strand et T. Thune (2003), la dimension technologique qualifie l'infrastructure technologique (matérielle et logicielle) mise en ouvre par une entreprise pour permettre à ses salariés d'accéder aux contenus e-learning. L'analyse des recherches ayant abordé cette thématique permet de relever deux composants centraux de l'infrastructure technologique : la plate-forme e-learning (M. Favier, 2003) et l'équipement informatique constitué du réseau (P. Henry, 2001; T. Lau, 2003 ; K. Young, 2001) et des ordinateurs (E. Bernardin, 2006).

\subsubsection{Dimension pédagogique: proposition d'une définition et présentation des attributs}

L'importance de la dimension pédagogique dans le déploiement de parcours e-learning a été soulignée par plusieurs auteurs (J.Lowyck, 2002 ; P. Tynjälä, P. Häkkinen, 2005; S. Vasquez Bronfman, 2004). Pour A. Welle-Strand et T. Thune (2003), la dimension pédagogique du déploiement recouvre les questions de "qui » suit le e-learning et de ce que suivent les apprenants (autrement dit, les contenus et activités pédagogiques qui leur sont proposés). R.E. Mayer (2003) précise que les modules multimédias d'autoformation peuvent être définis par le thème de leur contenu, les types d'activités multimédias qu'ils proposent et leur durée. E. Bernardin (2006) indique aussi que les formes d'interactions et les formes de contrôles permis dans un parcours e-learning caractérisent la dimension pédagogique. D'autres auteurs mettent l'accent sur la présence cognitive (S-M. Alavi et M. Taghizadeth, 2013) comme étant un élément essentiel à la réussite de la formation à distance. Dans le 
même sens, A. Jézérou (2010) distingue trois dimensions influant sur l'e-learning: la présence socio-cognitive, socio affective et pédagogique.

$\mathrm{Au}$ regard des éléments précédents, les dimensions du déploiement pédagogique sont les suivantes : les thèmes des contenus e-learning, les formes d'interactions et les formes de contrôles développés dans les parcours e-learning, ainsi que les activités pédagogiques possibles.

\subsubsection{Dimension organisationnelle : proposition d'une définition et présentation des attributs}

L'approche de A. Welle-Strand et T. Thune (2003) indique une troisième et dernière dimension à prendre en considération : l'organisation des parcours e-learning. Elles précisent que la dimension organisationnelle correspond à la question du "comment» : comment les modalités $e$-learning sont exposées aux salariés ? Selon nous, cette dimension caractérise les différentes manières dont un parcours e-learning est déployé vers des salariés. L'analyse des articles ayant abordé cet aspect permet de relever les aspects suivants : les modalités d'introduction des parcours e-learning auprès des salariés, les modes de connexion au e-learning, le lieu de suivi, l'éligibilité au Droit Individuel à la Formation (DIF) et les formes d'accompagnement.

\section{2. - Méthodologie de la recherche}

$\mathrm{Au}$ niveau méthodologique, quatre études qualitatives empiriques ont été conduites dans un grand groupe international spécialisé dans la conception, la fabrication et la commercialisation de véhicules automobiles, désigné "Construcauto » dans la suite de ce document. Trois raisons principales ont motivé le choix de ce terrain :

- l'investissement important et de longue date dans la formation par ce grand groupe ;

- l'intégration du e-learning dans son dispositif de formation ${ }^{1}$ depuis 1998 ;

- la multiplicité de ses parcours e-learning et des modes de déploiement mis en œuvre.

1. Selon le baromètre du Préau, beaucoup d'entreprises ont commencé à investir réellement dans le e-learning après 2005 . 
Le besoin de prendre en compte les spécificités des acteurs et de leur environnement a amené à privilégier une approche méthodologique qualitative par études de cas. Quatre études de cas enchâssées dans un même contexte organisationnel, ont été conduites. Elles correspondent à quatre parcours e-learning différents :

- processus logistiques dans l'entreprise ;

- PowerPoint ;

- efficacité personnelle ;

- et processus achats dans l'entreprise.

\subsection{Choix des études de cas}

«Le choix d'un cas unique peut se justifier par son caractère révélateur ; le choix de deux ou trois cas peut être légitimé par le caractère exploratoire d'une recherche... » (M. Hlady Rispal, 2002, p. 89). Ce travail de recherche se situe dans une approche quasi-exploratoire. Les recherches sur le e-learning sont en effet émergeantes. Elles se caractérisent par des fondements théoriques faibles et morcelés, et par un nombre limité de recherches conduites en entreprise (T. Bondarouk, H. Ruel, 2010, E. Bernardin, 2006 ; D.E.Leidner, S.L. Jarvenpaa, 1995). Le nombre théorique «idéal » d'études de cas oscille donc entre deux et trois. Nous sommes partis sur la base de deux études après la phase exploratoire terrain de notre étude de cas. Plusieurs autres possibilités se sont présentées au fur et à mesure de notre présence sur le terrain. Celles-ci nous permettaient de prendre en compte des parcours e-learning différents avec des modes de déploiement spécifiques. Le parcours e-learning logistique permet d'étudier un thème de formation métier qui porte sur les processus spécifiques de fonctionnement de l'entreprise, c'est-à-dire des savoirs à la fois d'environnement et procéduraux. Ce parcours a été déployé selon deux modes: en libre accès et par implication du supérieur hiérarchique ${ }^{2}$. Cette étude de cas met particulièrement l'accent sur le rôle spécifique du supérieur hiérarchique. Le parcours e-learning PowerPoint permet d'étudier un parcours de formation lié à la bureautique, autrement dit des savoir-faire, et le cadre spécifique d'un déploiement en centre de ressources. Le parcours efficacité personnelle permet d'étudier un parcours de formation lié au développement de savoir-être. Il a été déployé de deux manières différentes : en libre accès (tout e-learning) et en mixte (c'est-à-dire intégré à des séquences de formation en présentiel). Ce parcours

2. Le service formation et/ou le DRH de l'entité passe(nt) dans ce cas par les managers pour leur demander d'engager leur(s) collaborateur(s) dans le parcours e-learning. 
permet d'étudier plus spécifiquement l'influence du déploiement mixte. Le quatrième parcours e-learning porte sur les processus achats de l'entreprise et donc des savoirs d'environnement et procéduraux. C'est le dernier à avoir été développé dans l'entreprise lors de notre étude terrain. Il a été déployé de deux manières : en libre accès et en mixte. Il présente plusieurs spécificités. Il a tout d'abord été déployé auprès d'autres salariés que ceux utilisant quotidiennement dans leur travail ce type de connaissance. Au niveau du déploiement, une attention particulière a été portée à la qualité graphique. Le déploiement récent de ce parcours a en outre impliqué une présence plus importante du service formation, notamment dans le mode libre accès. Cette implication n'a pas toujours été neutre dans l'acte de suivi des salariés.

\subsection{Conduite des entretiens}

$\mathrm{Au}$ total, 81 entretiens ont été conduits dans le cadre de cette recherche. En premier lieu, 13 entretiens ont été menés lors de la phase pré-exploratoire avec des responsables de formation, responsables e-learning, salariés et formateurs de différentes entreprises. L'objectif était de mieux comprendre le contexte de la formation et du développement du e-learning en France, ainsi que les questions que soulevait l'intégration de la formation en ligne dans les dispositifs de formation. Ensuite, 20 entretiens ont été menés avec des membres de l'équipe projet e-learning et des responsables de formation de l'entreprise Construcauto lors de la phase exploratoire. L'enjeu était de mieux comprendre les modes de déploiement du e-learning dans cette multinationale et de s'imprégner des questions que générait le recours à cet ensemble de modalités de formation. Enfin, 48 entretiens, dont quatre avec des responsables de formation, ont été réalisés avec des salariés dans les quatre études de cas (soit en moyenne 11 entretiens par étude de cas). 
Tableau 1 : Utilisation des entretiens non-directifs et semi-directifs lors des trois phases de la recherche

\begin{tabular}{|l|c|c|c|}
\hline & $\begin{array}{c}\text { Nombre d'entre- } \\
\text { tiens avec des } \\
\text { responsables } \\
\text { formations et } \\
\text { e-learning }\end{array}$ & $\begin{array}{c}\text { Nombre } \\
\text { d'entretiens } \\
\text { avec des salariés }\end{array}$ & Nombre total \\
\hline $\begin{array}{l}\text { Phase } \\
\text { pré-exploratoire }\end{array}$ & 8 & 5 & 13 \\
\hline $\begin{array}{l}\text { Phase } \\
\text { exploratoire }\end{array}$ & 15 & 5 & 20 \\
\hline Phase intensive & 4 & 44 & 48 \\
\hline
\end{tabular}

Il est important de noter que tous les salariés interrogés ont réellement été engagés dans leurs parcours e-learning. Cela signifie qu'ils se sont connectés aux modules multimédias de leur parcours et les ont suivis. Six thèmes principaux ont été abordés, avec plusieurs thèmes de relance, au cours des entretiens :

- Parcours professionnel du salarié rencontré

- Enumération des formations et parcours e-learning suivis dans l'entreprise

- Processus d'entrée dans le parcours e-learning étudié

- Ressenti sur le déroulement du parcours e-learning étudié

- Apport(s) potentiel(s) du parcours e-learning étudié

- Comparaison entre le e-learning et la formation traditionnelle

\subsection{Analyse des entretiens}

Le processus d'analyse suivant a été suivi tout au long du travail :

- Phase 1 : rédaction de synthèse à la fin de chaque entretien.

- Phase 2 : rédaction de synthèse durant la phase de retranscription des données.

- Phase 3 : analyse d'entretiens pilotes et rédaction d'un dictionnaire des thèmes.

- Phase 4 : analyse systématique des entretiens avec NVivo.

- Phase 5 : génération de présentation sous forme de matrices et de tableau.

La structure du dictionnaire des thèmes a permis d'une part, d'isoler les dimensions caractéristiques d'un déploiement: dimension technologique, dimension pédagogique et dimension organisationnelle. D'autre part, ce dictionnaire a été organisé pour étudier l'influence de ces dimensions sur l'engagement des salariés. Quatre dimensions principales ont été étudiées : influence de l'environnement 
technologique, influence de l'environnement social, influence de l'environnement spatial et temporel. Le codage par Nvivo a permis de générer des matrices permettant d'étudier le rôle joué par l'environnement technologique, social, spatial et temporel sur l'engagement de l'ensemble des salariés interrogés.

\section{3. - Présentation des résultats}

L'analyse des entretiens a permis de mettre en exergue l'influence de l'environnement technologique, l'environnement social et l'environnement spatial et temporel sur l'engagement des salariés dans des parcours e-learning. Ces différents aspects sont analysés dans les paragraphes suivants avant qu'une mise en relation avec les différentes dimensions du déploiement ne soit réalisée.

\subsection{Influence de l'environnement technologique sur l'engagement des salariés}

Le rapport des salariés à l'environnement technologique d'un parcours e-learning peut être caractérisé par trois notions: la perception de l'utilité, de la facilité d'utilisation et de la qualité.

L'utilité perçue correspond à l'intérêt ressenti par les salariés de la modalité e-learning pour leur permettre d'apprendre sur une thématique donnée. Deux études de cas extrêmes de cette recherche peuvent être citées sur ce point : PowerPoint et efficacité personnelle. Dans la première étude de cas, la majorité des salariés ont indiqué que le e-learning leur semblait être un excellent moyen de se former sur ce logiciel de bureautique. Ils ont en effet indiqué que le e-learning constituait une forme de simulation proche de la réalité de l'utilisation du logiciel. Ce constat a même été reconnu par des salariés peu favorables au e-learning d'une manière générale. Dans la deuxième étude de cas, efficacité personnelle, quelques salariés ont exprimé des interrogations quant à l'intérêt de se former sur une thématique qui nécessite à leur sens d'être confronté à soi-même par l'expérience et les interactions avec les autres (formateurs, collègues). La majorité des salariés rencontrés a indiqué dans ce cas que le e-learning était un moyen d'apprendre des définitions, de conceptualiser quelques éléments, mais qu'il n'était en aucune manière une modalité de formation efficace pour apprendre sur cette thématique. Globalement, et à l'exception de quelques salariés dans l'étude de cas efficacité personnelle, les salariés ont souligné un intérêt du e-learning par rapport à leur thématique d'apprentissage. 
La facilité d'utilisation perçue correspond à la perception qu'ont les salariés de l'utilisation sans effort et sans difficulté des modules multimédias d'autoformation constitutifs d'un parcours e-learning. L'ensemble des études de cas a permis de relever que cette facilité d'utilisation s'exprime par un accès sans problème aux modules multimédias du parcours e-learning et à la facilité de navigation dans un module multimédia donné (accéder à la séquence suivante, actionner les jeux et les vidéos proposés, ...).

La qualité perçue exprime la satisfaction des salariés à l'égard de l'ergonomie des modules multimédias d'un parcours e-learning, de son contenu, et/ou de sa durée. L'étude de cas sur le parcours $e$ learning achat en particulier a permis de montrer l'importance de cette perception dans l'engagement. L'ergonomie de ce module a en effet fait l'objet d'un travail important pour rendre la formation conviviale. Le responsable formation a ainsi choisi de créer un univers extraterrestre pour engager les salariés dans leur formation. Ce but a été atteint au vu de l'envie suscitée par l'environnement de continuer à effectuer le parcours e-learning pour connaître la suite comme l'ont exprimé plusieurs salariés.

La quasi-totalité des salariés a exprimé un rapport positif à l'environnement technologique, quels que soient les parcours $e$ learning étudiés dans cette recherche.

\subsection{Influence de l'environnement social sur l'engagement des salariés}

L'analyse des quatre études de cas a permis de faire ressortir quatre sources potentielles d'interactions d'un salarié avec son environnement social :

- le service formation ;

- le supérieur hiérarchique ;

- les collègues ;

- et le formateur.

L'apparition des sources d'interactions est en partie dépendante du type de déploiement mis en œuvre. Un déploiement par l'implication du supérieur hiérarchique renforce ainsi l'apparition du rôle de celui-ci. La variété des formes d'incidence de l'environnement social sur les salariés, quelle que soit la source d'interaction, nous semble très intéressante. L'analyse des quatre parcours e-learning a fait ressortir trois modalités différentes de l'influence de l'environnement social sur l'engagement des salariés. L'environnement social peut ainsi être perçu comme incitatif, comme un soutien à l'engagement ou encore comme entravant. 
L'environnement social peut être décrit comme incitatif lorsque les salariés répondent par l'obéissance à un ordre hiérarchique ou lorsque leur acte est l'expression d'un conformisme aux normes sociales. Le parcours e-learning logistique est très instructif sur ce plan. L'implication du Directeur des Ressources Humaines du département et des responsables hiérarchiques directs a permis de relever plusieurs phénomènes d'obéissance à l'autorité. L'orientation «nouvelle technologie» de ce département, initiée par son ancien directeur, a favorisé l'émergence d'une norme sociale au sein des équipes. Une norme constitue une règle implicite à suivre. Il a été mis en évidence, à travers les entretiens conduits, qu'il est de bon ton de se montrer ouvert à l'utilisation des technologies de l'information et de la communication. Le parcours e-learning achat a pour sa part apporté des informations sur les formes de soutien. Le service formation s'est montré très présent dans le déploiement de ce parcours e-learning. Une forme de soutien informationnel a pu être relevée : la diffusion de l'information auprès des salariés quant à la disponibilité du parcours. Une forme de soutien émotionnel, à savoir l'accompagnement des salariés au cours de leur parcours, a aussi été constatée. Cela rejoint l'idée de A. Daele (2006) selon laquelle le formateur joue un rôle «facilitateur» via la coordination, l'animation et la modération, ce qui permet à l'apprenant à distance de bien assimiler la formation.

Il est intéressant de noter que ces modalités n'apparaissent pas de la même manière selon les modes de déploiement organisationnel choisis ; ceux-ci créent des environnements sociaux différents. Le mode de déploiement libre accès se distingue par un environnement social de soutien. Sans faire disparaître systématiquement ce premier aspect, le mode de déploiement encadré se caractérise principalement par son aspect social incitatif. Plusieurs salariés, notamment réticents ou peu favorables au e-learning, ont souligné l'importance d'un accompagnement social incitatif sur leur acte de suivi.

\subsection{Influence de l'environnement spatial et temporel sur l'engagement des salariés}

L'influence de l'environnement spatial et temporel s'est exprimée sur deux dimensions principales dans les quatre études de cas : le lieu et la période de formation. Trois modalités de rapport des salariés aux deux dimensions de l'environnement spatial et temporel ont été identifiées.

L'environnement spatial et temporel peut ainsi être décrit par les salariés comme facilitant ou à l'opposé entravant. Plusieurs salariés ont par ailleurs indiqué être à l'aise avec leur environnement spatial 
et/ou temporel, en se les appropriant pour organiser le suivi de leur parcours e-learning. L'environnement spatial et temporel peut dans ce cas de figure être décrit comme maîtrisé. Les salariés changent alors de lieu pour réaliser leur(s) module(s) multimédia(s) ou n'ont aucune difficulté à se former en open-space. Ils sont également capables de faire évoluer leur agenda pour y glisser les séances de suivi du e-learning.

L'étude de cas PowerPoint a été particulièrement intéressante par rapport à ces éléments, car les salariés ont tous souligné le caractère facilitateur de l'environnement dans lequel ils ont été amenés à suivre les modules e-learning. L'une des spécificités de cette étude de cas est le fait que le parcours e-learning soit présentialisé. Cela signifie que les salariés suivent les modules multimédias d'autoformation dans un lieu (centre de ressources) et pendant des périodes déterminés.

\subsection{Dimensions du déploiement, liens avec l'environnement technologique, social, spatial et temporel, et engagement}

En résumé, L'environnement technologique constitue un ensemble de facteurs invariants quels que soient les salariés, les parcours e-learning et leurs modes de déploiement. L'utilité perçue, la facilité d'utilisation perçue et la qualité perçue sont des facteurs incontournables, selon les salariés, pour expliquer leur engagement. L'environnement social influe de manière différente sur l'engagement des salariés. Cette variation dépend des contextes de déploiement et des individus. Différentes sources constituent cet environnement social : le supérieur hiérarchique, le service formation, les collègues et/ou le formateur. Les salariés ont besoin d'une influence différente de ces sources: incitation, soutien social informationnel, soutien social émotionnel selon leur attitude à l'égard du e-learning. L'environnement spatial et temporel, c'est-à-dire le lieu de formation et la période de formation, a également une influence différente sur l'engagement des salariés. Certains salariés s'accommodent très bien de l'espace et du temps pour réaliser leur parcours e-learning pendant que d'autres trouvent le lieu de formation et/ou la période de formation entravante pour suivre les modules multimédias de leur parcours e-learning.

$\mathrm{Au}$ niveau des facteurs d'engagement, le déploiement technologique joue sur la facilité d'utilisation et la qualité perçue par les salariés du dispositif technologique. Le déploiement pédagogique a une incidence sur la qualité perçue, notamment celle des contenus proposés, mais également sur l'utilité perçue, c'est-à-dire la capacité perçue par les salariés du e-learning de répondre à leur besoin 
d'apprentissage. Le déploiement organisationnel a une répercussion sur l'environnement social et l'environnement spatial et temporel. Le mode d'entrée des salariés, le service support qui leur est proposé, l'implication des supérieurs hiérarchiques de l'entreprise, des services formation, des formateurs sont autant de facteurs qui constituent l'environnement social. Les lieux de formation et les périodes de déploiement jouent pour leur part sur l'environnement spatial et temporel.

\section{Conclusion}

Les modes de déploiement du e-learning concourent à créer des environnements favorables à l'engagement des salariés selon leurs trois dimensions : technologique, pédagogique et organisationnelle. Ils créent en effet des configurations technologiques, humaines, spatiales et temporelles spécifiques susceptibles de favoriser ou non l'engagement dans des parcours e-learning par les salariés. Les dimensions technologique et pédagogique du déploiement assurent le socle des conditions favorables à l'engagement des salariés. Ces dimensions peuvent être pensées et mises en place de manière industrielle, c'est-à-dire répliquées à grande échelle, car elles répondront à la majorité des profils des salariés que ce soit sur les facteurs de suivi ou sur les apprentissages. L'environnement social, généré par les dimensions organisationnelle et pédagogique du déploiement, permet de prendre en compte la diversité des salariés et d'adapter les parcours à leurs profils, en proposant un accompagnement de soutien (principalement informatif) et/ou incitatif. Le déploiement organisationnel et pédagogique, à travers l'environnement social, permet ainsi l'individualisation des parcours e-learning aux salariés.

Deux évolutions dans le domaine du e-learning nous semblent intéressantes à analyser quant à leur impact sur l'engagement des salariés :

1. Au niveau technologique et pédagogique, il est intéressant de noter que le développement des serious games est susceptible de favoriser l'engagement des salariés. Cette approche pédagogique offre en effet des environnements virtuels de plus en plus réalistes pour la formation des salariés en entreprise.

2. Au niveau technologique, le développement du nomadisme dans la formation via le développement de l'utilisation des Smartphones et des tablettes pose également la question de son 
influence sur l'engagement des salariés. La disponibilité en tout temps et en tout lieu de certains contenus de formation favorise-telle l'envie d'apprentissage des cadres nomades durant leurs temps morts?

\section{RÉFÉRENCES BIBLIOGRAPHIQUES}

Alavi S M., Taghizadeth M., 2013, «Cognitive Presence in a Virtual Learning Community: An EFL Case ». Journal of Distance Education. Vol 27, 1.pp. 1-11.

BACHIMONT B. et al. 2002, Le procédé Scenari : une chaîne éditoriale pour la production de supports numériques de formation. Actes du colloque TICE 2002.

BAUJARD C., 2004, «Stratégies d'adoption e-learning et pratiques de formation de grandes entreprises ", Systèmes d'Information et Management, vol. 9, 4, pp. 31-46.

BAUJARD C., 2005, "Vers une meilleure compréhension du processus d'adoption de l'e-learning : l'intégration du projet organisationnel au contexte de formation ", In. Kalika M. et al. (Eds), e-RH : Réalités managériales, Vuibert, pp. 223-237.

BAUJARD C., 2007, "Management de la formation e-learning des entreprises », Revue Française de Gestion, vol. 177, pp. 21-34.

BAUJARD C., 2008, Stratégie e-learning, Hermes Lavoisier.

BECKETT D. et al., 2002, "Just-in-time training: techne meets phronesis», Journal of Workplace Learning, vol. 14, 8, pp. 332-339.

Bernardin E., 2006, "Efficacité de l'e-learning en entreprise : comparaison de deux environnements d'apprentissage chez Air France». Thèse en sciences de gestion, Université de Nantes.

Beamish N., Armistead C., Watkinson, M. et Armfiled G., 2002, «The development of e-learning in UK/European corporate organizations ", European Business Journal, Vol. 14, 3, pp. 105-15.

Bondarouk T., Ruel H., 2010, «Dynamics of e-learning: theoretical and practical perspectives Introduction to special issue », International journal of training and development, vol. 14, 3, pp. 149-154.

Clark R. C., MAYER R.E., 2008, E-learning and the science of multimedia learning, Second Edition, Pfeiffer.

Daele A., 2006, Animation et modération des communautés virtuelles d'enseignants. Dans Daele A., et Charlier B. (Dir.).Comprendre les communautés virtuelles d'enseignants: pratiques et recherches (p. 1526). Paris, France : L'Harmattan.

DEROUIN R. E. et al., 2005, «E-learning in organizations », Journal of Management Information Systems, vol. 31, 6, pp. 920-940. 
DRILlON D., 2002, "Transformation et rupture dans l'enseignement supérieur ? De l'enseignement traditionnel vers le "e-learning" », Gestion 2000, pp. 263-280.

FAVIER M., 2003, " Des questions autour du e-learning », In. Caron-Fasan M-L., Lesca N. (Eds), Présent et Futurs des systèmes d'information, Presses Universitaires de Grenoble, pp. 111-151.

Favier M., Kalika M., et Trahand J., 2004, «E-learning, E-formation : implications pour les organisations", Systèmes d'Information et Management, vol. 9,4, pp. 3-10.

GIL P., 2000, E-formation : NTIC et reengineering de la formation professionnelle. Edition Dunod.

FranKOLA K., 2001, «Why Online Learners Drop Out », Workforce, pp. 5360.

Henry P., 2001, « E-learning technology, content and services », Education + Training, vol. 43, 4/5, pp. 249-255.

Hlady Rispal M., 2002, Les Etudes de cas. Application à la recherche en gestion, de Boeck.

JeREZOU A., 2012. "La présence en e-learning: modèle théorique et perspectives pour la recherche». Revue de l'Éducation à Distance. Vol 26,1 .

KALIKA M., 2001, Le e-learning et la formation continue au management, FNEGE.

LAU T., 2003, « Developing an e-learning strategy: a corporate perspective », World Conference on E-Learning in Corporate, Government, Healthcare, and Higher Education (ELEARN), Phoenix, Arizona, USA.

LEIDNER D. E., JARVENPAA S. L., 1995, « The use of information technology to enhance management school education: A theoretical view», MIS Quarterly, vol. 14, 1, pp. 265-291.

LowYCK J., 2002, «Pedagogical Design », In. Adelsberger H. H. et al. (Eds), Handbook of Information Technologies for Education and Training, Springer, pp. 199-217.

LytRAS M. D., POuloudi A., 2001, «E-learning: just a waste of time», Seventh Americas Conference on Information systems, Boston.

MARChAND L., LAUZON N., 2004, " L'apprentissage en ligne en milieu manufacturier : une valeur ajoutée ? », Gestion, vol. 29, 1, pp. 59-68.

MARTIN G. et al., 2003, "When absorptive capacity meets institutions and (e)learners: adopting, diffusing and exploiting e-learning in organizations ", International Journal of Training and Development, vol. 7, 4, pp. 228-244.

MAYER R. E., 2003, Multimedia Learning, Cambridge University Press

Mitchell A., HonORE S., 2007, « Criteria for successful blended learning », Industrial and commercial Training, vol. 39, 3, pp. 143-149.

Mueller D., Strohmeier S., 2010, «Design characteristics of virtual learning environments: an expert study», International Journal of training and development, vol. 14, 3, pp. 209-222. 
NetTeland G., 2009, «Implementation of E-learning in a large organization: The critical role of relevance to work ", International Journal of Advanced Corporate Learning, vol 2, 3, pp. 58-65.

NEWTON D. et al., 2002, «Effective implementation of online learning: a case study of the Queensland mining industry », Journal of Workplace Learning, vol. 14, 4, pp. 156-165.

PicCOLI G. et al., 2001, "Web-based virtual learning environments: a research framework and a preliminary assessment of effectiveness in basic IT skills training », MIS Quarterly, 25, 4, pp. 401-426.

PRAT M., 2008, E-learning. Réussir un projet : pédagogie, méthodes et outils de conception, déploiement, évaluation, Editions ENI.

SALAS E. et al., 2002, "Emerging themes in distance learning research and practice: some food for thought », International Journal of Management Reviews, vol. 4, 2, pp. 135-154.

SAVALl H., ZARDET V. 2003, Maîtriser les coûts et les performances cachés, Economica, Paris.

TAYLOR A-S A., BACKLUND P. et NiKLASSON L. 2012, « The coaching cycle: A coaching by gaming approach in Serious Game», Simulation and Gaming, vol 43(5), pp. 648-672.

TYNJÄLÄ P., HÄKKINEN P., 2005, «E-learning at work: theoretical underpinnings and pedagogical challenges », Journal of Workplace Learning, vol. 17, 5/6, pp. 318-336.

Upadhyaya K.T., Debasis, M., 2013. «E-learning as a Social-Technical System: An Insight intro factors influencing effectiveness », Business Perspectives \& Research, vol 2, 1, pp. 1-12.

VASQUEZ BRONFMAN S., 2004, «Facteurs de succès dans la mise en œuvre de projets e-learning : le cas d'une banque », Systèmes d'Information et Management, vol. 9, 4, pp. 47-61.

Welle-Strand A., Thune T., 2003, «E-learning policies, practices and challenges in two Norwegian organizations ", Evaluation and Program Planning, vol. 26, 2, pp. 185-192.

WeLSH E. T. et al., 2003, «E-learning: emerging uses, empirical results and future directions », International Journal of Training and Development, vol. 7, 4, pp. 245-258.

WILD R. H. et al., 2002, « A framework for e-learning as a tool for knowledge Management», Industrial Management and Data Systems, vol. 102, 7, pp. 371-381.

Young K., 2001, «The effective deployment of e-learning », Industrial and Commercial Training, vol. 33, 1, pp. 5-11. 